\title{
Time frequency and array processing of non-stationary signals
}

\author{
Adel Belouchrani ${ }^{1 *}$, Karim Abed-Meraim² ${ }^{2}$ and Boualem Boashash ${ }^{3,4}$
}

\section{Introduction and overview}

Conventional time-frequency analysis methods were extended to data arrays in many applications, and there is the potential for more synergistic development of new advanced tools by exploiting the joint properties of timefrequency methods and array signal processing methods. Conventional array signal processing assumes stationary signals and mainly employs the covariance matrix of the data array. This assumption is motivated by the crucial need in practice for estimating sample statistics by resorting to temporal averaging under the additional hypothesis of ergodic signals. When the frequency content of the measured signals is time varying (i.e., non-stationary signals), this class of approaches can still be applied. However, the achievable performances in this case are reduced with respect to those that would be achieved in a stationary environment. Instead of considering the nonstationarity as a shortcoming, time frequency array processing (TFAP) takes advantage of the nonstationarity by considering it as a source of information in the design of efficient algorithms in such nonstationary environments. Generally, this significantly improves performance. This improvement comes essentially from the fact that the effects of spreading the noise power while localizing the source energy in the time frequency domain increases the signal to noise ratio (SNR). Such approaches found applications in many important fields dealing with nonstationary signals and multi-sensor systems, such as biomedical, radar, seismic, telecommunications, and mechanical engineering.

\section{Specific advances}

This special issue focuses on this synergistic relationship between time-frequency methods and array signal processing methods and addresses recent developments, advances, and ideas in the field of time frequency and array processing of nonstationary signals with special emphasis given to their applications. There were 30 articles submitted to this special issue. The review process selected 15 accepted articles from the submissions.

In the article "Joint DOD/DOA estimation in MIMO radar exploiting time-frequency signal representations" [1] Yimin Zhang et al. deal with the joint estimation of direction-of-departure (DOD) and direction-ofarrival (DOA) information of maneuvering targets in a bistatic multiple-input multiple-output (MIMO) radar system when exploiting spatial time-frequency distribution (STFD). STFD has been successfully used in solving various array processing problems, such as direction finding and blind source separation, where nonstationary signals with time-varying spectral characteristics are encountered. The use of STFD is extended to MIMO radar systems. It is then demonstrated that the STFD is an effective tool in MIMO radar processing when moving targets produce Doppler signatures that are highly localized in the time-frequency domain.

In the article "Estimating the number of components of a multicomponent nonstationary signal using the shortterm time-frequency Rényi entropy" [2], Victor Sucic et al. propose a solution to the problem of detecting the local number of signal components by resorting to the shortterm Rényi entropy of signals in the time-frequency plane. The method does not require any a priori information about the analyzed signal, nor the knowledge of the Rényi entropy of one of the signal components. This method has been successfully applied to both synthetic and real life signals. Note that such approach is of particular interest in the selection of the time frequency points (signal autoterms) needed in the spatial time frequency distribution (STFD) based array signal processing.

\footnotetext{
*Correspondence: adel.belouchrani@enp.edu.dz

1 Electrical Engineering Department, Ecole Nationale Polytechnique, Algiers, Algeria

Full list of author information is available at the end of the article
}

\section{照 Springer}

(c) 2012 Belouchrani et al. licensee Springer. This is an Open Access article distributed under the terms of the Creative Commons Attribution License (http://creativecommons.org/licenses/by/2.0), which permits unrestricted use, distribution, and reproduction in any medium, provided the original work is properly cited. 
In the article "Performance analysis for time-frequency MUSIC algorithm in presence of both additive noise and array calibration errors" [3] Mohamed Khodja et al. present a comparative analytic performance analysis of the Time frequency MUSIC algorithm that uses STFDs with respect to the conventional MUSIC algorithm that uses the data covariance matrix when the received array signals are subject to calibration errors in a non-stationary environment. An unified analytical expression of the direction of arrival (DOA) error estimation is derived for both methods. It is shown that for low signal to noise ratio (SNR) and high signal to sensor perturbation ratio (SPR) the STFD method gives better performance, while for high SNR and for the same SPR both methods give similar performance.

In the article "An efficient joint estimation of wideband polynomial-phase signal parameters and directionof-arrival in sensor array" [4], Igor Djurović et al. deal with the joint estimation of the direction-of-arrival (DOA) and parameters of wideband polynomial-phase signals (PPSs) in sensor arrays. Particular attention is given to secondand third-order PPSs. The developed estimation algorithm shows to be computationally efficient when applied in the context of large antenna arrays. It is also reported that the variance of the PPS highest order parameters reaches the Cramer-Rao lower bound (CRLB), while the DOA variance is above the CRLB for around $1 \mathrm{~dB}$ (for second-order PPS) and around $6 \mathrm{~dB}$ (for third-order PPS).

In the article "Blind source separation for robot audition using fixed HRTF beamforming" [5] Mounira Maazaoui et al. propose a two-stage blind source separation (BSS) algorithm for robot audition. The first stage consists of a fixed beamforming preprocessing to reduce the reverberation and the environmental noise. The beamforming filters are estimated by using the pre-measured robot head related transfer functions (HRTFs). The second stage is a BSS algorithm based on a sparsity criterion consisting of the minimization of the 11 norm of the sources. Different configurations of the proposed algorithm are presented. Promising results are provided showing separation improvement when using the fixed beamforming preprocessing.

In the article "Filtering in the joint time/chirp-rate domain for separation of quadratic and cubic phase chirp signals" [6], Mehmet Özgen proposes a linear time/chirprate (TCR) filter for decomposing multicomponent signals into their quadratic and/ or cubic phase chirp components. The article shows that it is easier to design filter masks in the TCR domain than in the time-frequency domain for recovering downsampled cubic phase signals and resolving back-to-back objects from in-line holograms.

In the article "Efficient phase estimation for the classification of digitally phase modulated signals using the
cross-WVD: a performance evaluation and comparison with the $S$-transform" [7] Chee Yen Mei et al. present an algorithm based on the cross-Wigner-Ville distribution (XWVD) for optimum phase estimation within the class of phase shift keying signals. An adaptive window kernel is proposed where the window is adjusted using the localized lag autocorrelation function. It is shown that the adaptive windowed XWVD (AW-XWVD) based phase estimator meets the Cramer-Rao lower bound (CRLB) at signal-to-noise ratio (SNR) of $5 \mathrm{~dB}$ for both binary phase shift keying and quadrature phase shift keying. Compared to the $S$-transform, the proposed AW-XWVD presents better performance.

In the article "Dereverberation and denoising based on generalized spectral subtraction by multi-channel LMS algorithm using a small-scale microphone array" [8], Longbiao Wang et al. propose a blind dereverberation method based on generalized spectral subtraction (GSS) in a multi-sensor system. The missing feature theory (MFT), initially proposed for enhancing the robustness to additive noise, is extended to dereverberation. This has lead to a one-stage dereverberation and denoising GSS based method that simultaneously suppress both the additive noise and nonstationary multiplicative noise (reverberation). Experimental results show that the proposed method is robust in a variety of reverberant environments for both isolated and continuous speech recognition.

In the article "Compressive sampling of swallowing accelerometry signals using time-frequency dictionaries based on modulated discrete prolate spheroidal sequences" [9], Ervin Sejdić et al. propose compressive sensing (CS) algorithm for accurate reconstruction of dual-axis swallowing accelerometry signals from sparse samples when monitoring physiological functions. The proposed algorithm uses a time-frequency dictionary based on modulated discrete prolate spheroidal sequences (MDPSS). The proposed CS algorithm is successfully applied on both synthetic test signals and swallowing accelerometry signals.

In the article "Dempster Shafer fusion of multisensor signals in nonstationary Markovian context" [10] Mohamed El Yazid Boudaren et al. propose a new approach to model multisensor nonstationary signals in the Markovian context. The proposed model allows to benefit simultaneously from both the Markov theory and theory of evidence. For this purpose, Dempster Shafer combination rule is used in order to take into account the nonstationary aspect of the hidden data of interest, and to fuse the different sensor signals in the Markovian context to improve segmentation accuracy. Experimental results demonstrated the effectiveness of such modeling with respect to conventional methods. 
In the article "Independent vector analysis based on overlapped cliques of variable width for frequencydomain blind signal separation" [11] Intae Lee et al. propose a method to improve the performance of independent vector analysis (IVA) for blind signal separation of acoustic mixtures. IVA is a frequency-domain approach that successfully resolves the well-known permutation problem by applying a spherical dependency model to all pairs of frequency bins. The proposed method allows variable amounts of statistical dependencies according to the correlation coefficients observed in real life acoustic signals and enables more accurate modeling of statistical dependencies. Application to speech signal show improved performances over conventional IVA.

In the article "Time-varying bispectral analysis of visually evoked multi-channel EEG" [12] Vinod Chandran exploits time-varying bispectral for the analysis of evoked EEG responses to flash visual stimulii. The use of EEG electrode array allows to obtain information about phase synchronization at different regions of the brain as the neural response develops.

In the article "Contribution of statistical tests to sparseness-based blind source separation" [13] Si Mohamed Aziz Sbai et al. propose two statistical tests for the reduction of the number of empirical parameters involved in standard sparseness-based underdetermined blind source separation (UBSS) algorithms. The first test performs multisource selection of the suitable timefrequency points for source recovery. The second one is dedicated to autosource selection for mixing matrix estimation. It is shown that the use of the proposed tests incurs no performance loss and improves the performance of standard weak-sparseness UBSS algorithms.

In the article "Evaluation of the modified $S$-transform for timefrequency synchrony analysis and source localization" [14] Said Assous et al. consider the problem of phase synchrony and coherence analysis using a modified version of the $S$-transform, referred to as the modified $S$ transform (MST). It allows the study of the phase coupling between two or more different spatially recorded entities with non-stationary characteristics. The proposed MST is applied to newborn EEG data and leads to improvements in resolution with respect to the standard $S$-transform.

In the article "A methodology for time-frequency image processing applied to the classification of nonstationary multichannel signals using instantaneous frequency descriptors with application to newborn EEG signals" [15] Boualem Boashash et al. present a general methodology for processing non-stationary signals for the purpose of classification and localization. The methodology combines methods adapted from three complementary areas: time-frequency signal analysis, multichannel signal analysis and image processing. It is referred to as multichannel time-frequency image processing. This new methodology is applied to the problem of classifying electroencephalogram (EEG) abnormalities in both adults and newborns.

\section{Conclusions}

The 15 articles included in this special session indicate that the field is alive and well, with a number of very promising directions covering a wide range of applications from Radar to Medicine. The editors hope that this special session will serve as further impetus to innovation in this exciting research field.

\section{Competing interests}

The authors declare that they have no competing interests.

\section{Acknowledgements}

We wish to acknowledge all the reviewers who have helped considerably to improve the quality of this special issue.

\section{Author details}

${ }^{1}$ Electrical Engineering Department, Ecole Nationale Polytechnique, Algiers, Algeria. ${ }^{2}$ Polytech'Orléans, University of Orléans, Orléans, France. ${ }^{3}$ The University of Queensland, Brisbane, QLD 4072, Australia. ${ }^{4}$ College of Engineering, Qatar University, Doha, Qatar.

Received: 16 October 2012 Accepted: 16 October 2012

Published: 30 October 2012

\section{References}

1. YD Zhang, MG Amin, B Himed, Joint DOD/DOA estimation in MIMO radar exploiting time-frequency signal representations. EURASIP. J. Adv. Signal Process. 2012, 102 (2012)

2. V Sucic, N Saulig, B Boashash, Estimating the number of components of a multicomponent nonstationary signal using the short-term time-frequency Rényi entropy. EURASIP J. Adv. Signal Process. 2011, 125 (2011)

3. M Khodja, A Belouchrani, K Abed-Meraim, Performance analysis for time-frequency MUSIC algorithm in presence of both additive noise and array calibration errors. EURASIP J. Adv. Signal Process. 2012, 94 (2012)

4. I Djurovic, S Djukanovic, M Simeunovic, P Rakovic, B Barkat, An efficient joint estimation of wideband polynomial-phase signal parameters and direction-of-arrival in sensor array. EURASIP J. Adv. Signal Process. 2012, 43 (2012)

5. M Maazaoui, K Abed-Meraim, Y Grenier, Blind source separation for robot audition using fixed HRTF beamforming. EURASIP J. Adv. Signal Process. 2012, 58 (2012)

6. M Özgen, Filtering in the joint time/chirp-rate domain for separation of quadratic and cubic phase chirp signals. EURASIP J. Adv. Signal Process. 2012, 122 (2012)

7. C Mei, A Sha'ameri, B Boashash, Efficient phase estimation for the classification of digitally phase modulated signals using the cross-WVD: a performance evaluation and comparison with the S-transform. EURASIP J. Adv. Signal Process. 2012, 65 (2012)

8. L Wang, K Odani, A Kai, Dereverberation and denoising based on generalized spectral subtraction by multi-channel LMS algorithm using a small-scale microphone array. EURASIP J. Adv. Signal Process. 2012, 12 (2012)

9. E Sejdic, A Can, LF Chaparro, CM Steele, T Chau, Compressive sampling of swallowing accelerometry signals using time-frequency dictionaries based on modulated discrete prolate spheroidal sequences. EURASIP J. Adv. Signal Process. 2012, 101 (2012)

10. MEY Boudaren, E Monfrini, W Pieczynski, A Ä̈ssani, Dempster-Shafer fusion of multisensor signals in nonstationary Markovian context. EURASIP J. Adv. Signal Process. 2012, 134 (2012)

11. I Lee, G-J Jang, Independent vector analysis based on overlapped cliques of variable width for frequency-domain blind signal separation. EURASIP J. Adv. Signal Process. 2012, 113 (2012)

12. V Chandran, Time-varying bispectral analysis of visually evoked multichannel EEG. EURASIP J. Adv. Signal Process. 2012, 140 (2012) 
13. SMA Sbai, AAE Bey, D Pastor, Contribution of statistical tests to sparseness based blind source separation. EURASIP J. Adv. Signal Process. 2012, 169 (2012)

14. S Assous, B Boashash, Evaluation of the modified S-transform for time-frequency synchrony analysis and source localisation. EURASIP J. Adv. Signal Process. 2012, 49 (2012)

15. B Boashash, L Boubchir, G Azemi, A methodology for time-frequency image processing applied to the classification of non-stationary multichannel signals using instantaneous frequency descriptors with application to newborn EEG signals. EURASIP J. Adv. Signal Process. 2012, 117 (2012)

doi:10.1186/1687-6180-2012-230

Cite this article as: Belouchrani et al:: Time frequency and array processing of non-stationary signals. EURASIP Journal on Advances in Signal Processing 2012 2012:230.

\section{Submit your manuscript to a SpringerOpen ${ }^{\circ}$ journal and benefit from:}

- Convenient online submission

- Rigorous peer review

- Immediate publication on acceptance

- Open access: articles freely available online

- High visibility within the field

- Retaining the copyright to your article

Submit your next manuscript at $\boldsymbol{~ s p r i n g e r o p e n . c o m ~}$ 